\title{
Effect of gestational age (preterm or full term) on lipid composition of the milk fat globule and its membrane in human colostrum
}

\author{
Antonio Pérez-Gálvez, ${ }^{1}$ María V. Calvo, ${ }^{2}$ Javier Megino-Tello, ${ }^{2}$ Josefa Aguayo-Maldonado, ${ }^{3}$ \\ Rafael Jiménez-Flores, ${ }^{4}$ and Javier Fontecha ${ }^{2 *}$ \\ ${ }^{1}$ Food Phytochemistry Department, Instituto de la Grasa (CSIC), Campus Universitario, Building 46, 41013, Sevilla, Spain \\ ${ }^{2}$ Food Lipid Biomarkers and Health Group, Institute of Food Science Research (CIAL, CSIC-UAM), 28049, Madrid, Spain \\ ${ }^{3}$ Neonatology Unit, Hospital Virgen del Rocío, 41013, Sevilla, Spain \\ ${ }^{4}$ Food Science and Technology Department, The Ohio State University, Columbus 43210
}

\begin{abstract}
Human colostrum is the first milk secreted by the mother after birth and constitutes the ideal food for the newborn, because its chemical composition, rich in immunoglobulins, antimicrobial peptides, growth factors, bioactive lipids, and other important molecules, is perfectly adapted to the metabolic, digestive, and immunological immaturity of the newborn. An incomplete gestational period can affect the maturity of the mammary gland and its ability to secrete milk with the proper composition for the newborn's condition. Previous studies indicate that the mammary gland modulates the profiles of bioactive lipids present in the different phases of lactation from colostrum to mature milk. Given the key role played by the polar lipids (PL) (phospho- and sphingolipids) of the milk fat globule membrane (MFGM) in the immune system and cognitive development of the newborn, it is crucial to analyze whether the content and distribution of the PL are affected by gestation period. Therefore, this study aimed to determine the milk fat globule (MFG) and MFGM lipid compositions of human colostrum samples from 20 healthy preterm and full-term mothers. Lipid characterization using chromatographic techniques (gas chromatograph mass spectrometry and HPLC-evaporative light-scattering detection) revealed differences related to length of gestation in the profiles of lipid classes and fatty acid and triacylglyceride contents of colostrum. This comparative analysis leads to noteworthy outcomes about the changing roles of the PL, considering the preterm or full-term condition. We found a lack of correlation of some PL (such as phosphatidylcholine, phosphatidylinositol, and phosphatidylserine) with the delivery term; these could be denoted as structural
\end{abstract}

Received February 25, 2020.

Accepted April 20, 2020.

*Corresponding author: j.fontecha@csic.es category lipids. However, sphingomyelin and phosphatidyl-ethanolamine exhibited trends to decrease in full-term colostrum, indicating that in the final stage of pregnancy specific accretion of some PL occurs, which should be denoted as a nutritional redistribution.

Key words: human colostrum, gestational age, milk fat globule membrane, polar lipid

\section{INTRODUCTION}

Several epidemiological and controlled trials support the current WHO recommendation of exclusive breastfeeding up to 6 mo of age as the most appropriate method of providing the newborn with a complete and diverse array of macro- and micronutrients, fulfilling the requirements of energy, growth factors, and bioactive compounds (Kramer and Kakuma, 2012). Among the high number of metabolic processes that have evolved to produce milk, the secretion of lipids stands out. Lipids are secreted in the form of organized colloidal macrostructures called milk fat globules (MFG), a process orchestrated in the secretory epithelial cells of the mammary glands, which are very active and have well-developed synthetic capabilities for lipids (McManaman and Neville, 2003; German and Dillard, 2006; McManaman et al., 2006). Lipids are synthetized in the smooth endoplasmic reticulum, where they accumulate as small lipid accretions in the gap between the inner and outer leaflets of this organelle. Then, the lipids are secreted as nanodroplets to the cytoplasm covered by a section of the outer leaflet of the endoplasmic reticulum. Subsequently, the lipid droplets tend to condense during transport to the apical plasma membrane, where they are secreted by a budding process and covered by a section of the cellular membrane (Mather, 2000). The main consequence of this process is that the milk fat globule membranes (MFGM) are structured as a trilayer of polar lipids (PL) and membrane-specific proteins (Keenan and Mather, 2006; Lopez, 2011), with 
a core of triacylglycerides, cholesteryl esters, and other minor apolar lipids that represent 95 to $98 \%$ of the MFG (Lopez et al., 2019; Ortega-Anaya and Jimenez-Flores, 2019). Consequently, the different lipid categories that encompass the MFG are segregated in 2 locations; that is, the apolar core and the polar envelope, which contains the original apical plasma membrane of the mammary epithelial cells. Hence, isolation of the MFGM and its subsequent analysis provide access to biologically relevant information regarding the asymmetry of membrane components in the outer bilayer and in the interior leaflet facing the core of the MFG (Deeth, 1997), the lateral organization of the membrane lipids into macro- and microdomains (Lopez and Ménard, 2011; Zou et al., 2015; Nguyen et al., 2016), and the identities, properties, and amino acid sequences of the membrane proteins (Mather, 2000). However, access to these characteristics of the MFGM is limited to the breastfeeding period that mammary tissues reach after pregnancy. Lipid composition of the MFG is dynamic over the course of lactation, reflecting factors associated with the mother, including diet and environment, maternal genetics, and even adaptation to the needs of the infant (German and Dillard, 2006; Cilla et al., 2016; Lee et al., 2018). Gestational age is recognized as a significant factor that influences the composition of some milk lipids, such as long-chain PUFA (Bitman et al., 1983; Bokor et al., 2007), PL (Shoji et al., 2006; Ingvordsen Lindahl et al., 2019), and other lipid categories (Xavier et al., 2018).

Although other studies suggest either a translational composition of the milk adapted to the preterm infant or an insufficient pattern of key components considering the condition in preterm birth, the cellular membrane of the mammary epithelium might be completely functional and operative during pregnancy, which may explain why differences in the lipid composition of the MFGM could appear with preterm delivery. Indeed, this interpretation would mean that the programmed metabolic activity for lactation intentionally includes an adaptation of the cellular membrane of the mammary epithelial cells as a nutrition source. A preterm delivery leads to an immature newborn and also to immature mammary glands, disrupting the regular process of lipid accretion, including the potential refurbishment process of the membrane of the epithelial cells denoted above. In this work, we aim to determine the effects of gestational age at birth in the different lipid compositions of the MFG and MFGM. We selected preterm and full-term human colostrum samples because it is at these stages of lactation where the qualitative and quantitative composition of lipids can most probably be ascertained.

\section{MATERIALS AND METHODS}

\section{Subjects}

The study population comprised 20 healthy women who were recruited within a 1-mo period in 2018 and 2019. The sample size was calculated according to Julious and Owen (2006). Mothers were classified in 2 groups: mothers who gave birth to full-term neonates (37 to $40 \mathrm{wk}$, single birth) and mothers who gave birth to preterm neonates (25 to $35 \mathrm{wk}$, single birth). Eligible participants in this study were nonsmoking mothers with no chronic diseases. Exclusion criteria applied were pathologies or infections during the gestation, developmental anomalies in the fetus, or death of the child. Mothers following any special diets, such as vegetarians or those taking any prenatal supplements, were not included. The volunteers provided informed consent to participate in the research program, and none of the initially enrolled mothers dropped out of the study.

\section{Materials and Reagents}

Dichloromethane, chloroform, hexane, methanol, isooctane, isopropanol, dimethylformamide, and acetonitrile, all of HPLC grade, were purchased from Labscan Ltd. (Dublin, Ireland). Sodium sulfate anhydrous and sodium carbonate were obtained from Panreac Química SLU (Barcelona, Spain). Methyl tert-butyl ether was supplied by VWR International Eurolab SL (Barcelona, Spain). Sodium methoxide (95\%), sodium citrate dehydrate, formic acid (98\%), triethylamine (99.5\%), the triacylglyceride (TAG) standards trinanoin and tritridecanoin, the free fatty acid standards, phosphatidylcholine (PC), phosphatidylinositol (PI), phosphatidylserine (PS), phosphatidylethanolamine $(\mathbf{P E})$, and sphingomyelin (SM) standards, as well as $5 \alpha$-cholestane and cholesterol (Chol) were supplied by Sigma-Aldrich (St. Louis, MO). Reference samples with known composition, such as butter fat BCR-164 and BCR-519 (EU Commission; Brussels, Belgium) were from Fedelco SL (Madrid, Spain).

\section{Samples}

Milk Collection. Samples were collected at the Neonatology Unit of the Hospital Universitario Virgen del Rocío (Seville, Spain). Preterm and full-term mothers donated colostrum at 3 to $5 \mathrm{~d}$ postpartum. Milk samples were obtained by collection of the total milk volume of 1 breast during 1 milk expression session into a polypropylene bottle. Milk collection was recom- 
mended in the morning 2 to $4 \mathrm{~h}$ after the previous breastfeeding. Samples were directly transported to the laboratory and stored at $-80^{\circ} \mathrm{C}$ until analysis.

Isolation of MFG and MFGM. The colostrum samples were separated into 2 aliquots. One was used for the isolation of the MFG fraction, which was achieved using the methodology published by Patton and Huston (1986). The resulting MFG fraction was stored at $-35^{\circ} \mathrm{C}$ until analysis. The second aliquot was used for isolation of MFGM (Calvo et al., 2020). Colostrum was mixed with $2 \%$ (wt/vol) sodium citrate dihydrate, and the mixture was incubated at room temperature for $1 \mathrm{~h}$. The incubated sample was centrifuged at 70,000 $\times g$ for $45 \mathrm{~min}$ at $15^{\circ} \mathrm{C}$ in a Beckman Optima MAX Ultracentrifuge (Beckman Coulter Inc., Indianapolis, IN). The resulting sample was kept in ice for $30 \mathrm{~min}$, and the supernatant was carefully discarded to avoid contamination of the pellet, which was washed with Milli-Q water (Millipore, Billerica, MA), freeze-dried, and stored at $-35^{\circ} \mathrm{C}$ until analysis.

Analysis of Lipid Categories. The methodologies applied for extraction of fat, determination of fat content, and analysis of lipid categories have recently been published by Calvo et al. (2020). Briefly, lipids were isolated from the MFG and MFGM fractions with an optimized solvent-to-sample amount (Löfgren et al., 2012). Separation of lipid classes by an HPLC-evaporative light-scattering detector (ELSD) was performed with an HPLC system (Model 1260, Agilent Technologies Inc., Palo Alto, CA) coupled to an ELSD (SEDEX 85 Model, Sedere SAS, Alfortville, France), as detailed by Castro-Gómez et al. (2014a). Samples and standards were analyzed under the same conditions, using freshly prepared solvents. Assays were carried out in triplicate. The determination of TAG and Chol by GC-flame ionization detector was performed on a Clarus 400 GC (PerkinElmer Ltd., Beaconsfield, UK) equipped with an automatic split/splitless injector and a flame ionization detector. The experimental chromatographic conditions were as published by Calvo et al. (2020). For qualitative and quantitative analysis of TAG, response factors were calculated using an anhydrous milk fat (reference material BCR-519) of known TAG and Chol composition, and glyceryl trinanoate as internal standard $(100 \mu \mathrm{L} ; 1 \mathrm{mg} / \mathrm{mL})$.

We prepared FAME using the direct transesterification method described by Castro-Gómez et al. (2014b). Tritridecanoine (C13:0-TAG) was used as an internal standard $(200 \mu \mathrm{L} ; 1.3 \mathrm{mg} / \mathrm{mL})$ and added to samples before methylation. To separate FAME, we used an Agilent chromatograph (model 6890N; Agilent Technologies Inc.) equipped with an MS detector (Agilent $5973 \mathrm{~N}$ ) and a CP-Sil 88 fused-silica capillary column $(100 \mathrm{~m} \times 0.25 \mathrm{~mm}$ internal diameter, $0.2 \mu \mathrm{m}$; Chrom- pack, Middelburg, the Netherlands). For qualitative and quantitative analysis, response factors were calculated using anhydrous milk fat (reference material BCR-164). All analyses were carried out in at least triplicate.

\section{Ethics Approval}

All volunteers gave informed consent for inclusion before they participated in the study, which was conducted in accordance with the Declaration of Helsinki (https://www.wma.net/policy/current-policies/). The study protocol was approved by the Ethics Committee of the Hospital Universitario Virgen del Rocío (AGL2017-87884R).

\section{Statistical Analysis}

Data are reported as the median, including 25th and 75 th percentiles. Due to the non-normality of content of lipids categories (Shapiro-Wilk test) and the lack of homoscedasticity (Levene test), the data were analyzed using a nonparametric statistical procedure in SPSS (version 25, IBM Corp., Armonk, NY). The Mann-Whitney test was applied to analyze differences in the contents of lipid categories of the MFG and the MFGM, considering the pair preterm and full-term samples. Comparisons were also made between data, independent of gestational age, considering the pair MFG and MFGM samples. Significance was set at $P$ $<0.05$.

\section{RESULTS AND DISCUSSION}

\section{Organization of Lipid Classes in MFG from Human Colostrum}

The MFG is a unique assembly, where lipid classes are segregated in 2 different locations: the core, where mostly TAG are found, and the protected surrounding envelope, which is the biological membrane of the mammary epithelial cells, composed of PL and membrane proteins, comprising an outstanding source of bioactive molecules (Keenan and Mather, 2006). To the best of our knowledge, this is the first study in which the former segregation of lipid categories has been analyzed in human colostrum samples isolating the MFG and their MFGM fractions separately.

Independent of gestational age, some lipid families were preferentially accumulated in 1 of the 2 locations of the colostrum fat. Considering the neutral lipid classes, the TAG fraction was highly represented in the MFG (93\%, Table 1), with the rest of the lipids in minor proportions, whereas the MFGM was both qualitatively and quantitatively heterogeneous in neutral 
Table 1. Organization of lipids classes in milk fat globule (MFG) and milk fat globule membrane (MFGM) from human colostrum at different gestational ages; data are reported as the median, with 25th and 75th percentiles, of the differences in content of lipid categories (\%) of the MFG and the MFGM ${ }^{1}$

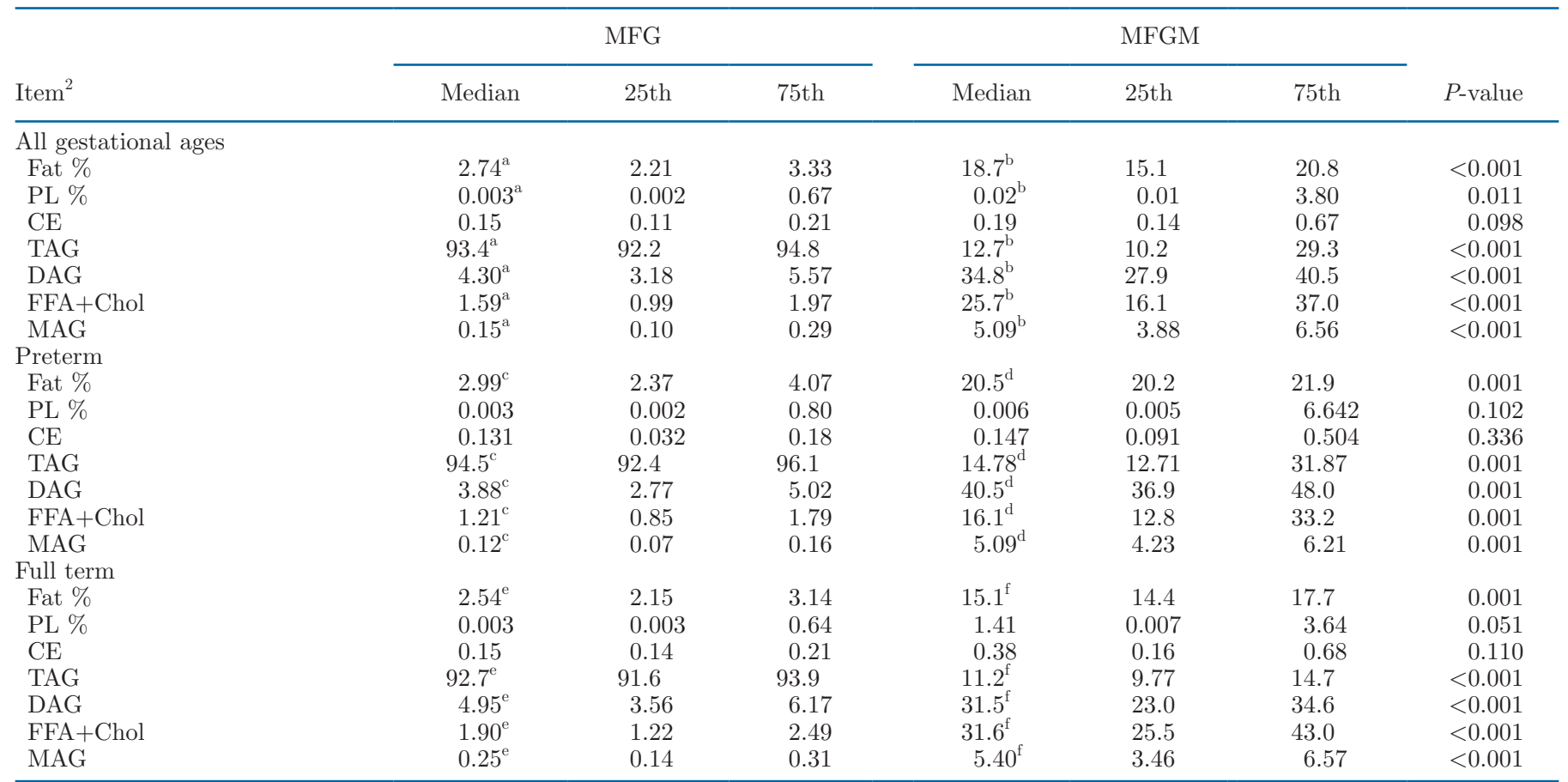

\footnotetext{
${ }^{\mathrm{a}, \mathrm{b}}$ Median values significantly different (MFG vs. MFGM) from samples studied independent of gestational age.

${ }^{\mathrm{c}, \mathrm{d}}$ Median values significantly different (MFG vs. MFGM) from preterm samples.

${ }^{\mathrm{e}, \mathrm{f}}$ Median values significantly different (MFG vs. MFGM) from full-term samples.

${ }^{1}$ Comparisons were performed for data determined in MFG and MFGM fractions from human colostrum samples. Significance was set at $P<$ 0.05 .

${ }^{2} \mathrm{PL}=$ polar lipids $; \mathrm{CE}=$ cholesterol esters; $\mathrm{TAG}=$ triacylglyceride DAG = diacylglyceride FFA + Chol = free fatty acids and cholesterol; $\mathrm{MAG}$ $=$ monoacylglyceride.
}

lipid classes. By contrast, TAG (12.7\%) was not the main lipid category in the MFGM. The diacylglyceride fraction $(34.8 \%)$, followed by free fatty acids plus Chol $(25.7 \%)$ and the monoacylglycerides (5\%), were significantly present in the MFGM (Table 1) compared with their presence in the lipid profile of the MFG $(4.3 \%, 1.6 \%$, and $0.15 \%$ for the diacylglyceride, free fatty acids plus Chol, and monoacylglycerides, respectively, Table 1). These data from colostrum fat were different from the data published by Jensen (1989), in which TAG was quantified as the major fraction in the MFGM from human milk, although it was not specified whether samples were colostrum or mature milk. In this study, we performed analysis of the chain length distribution by carbon atom number (CAN) of the TAG and observed a distinct pattern depending on the fat location where the TAG reside (Figure 1A). The pattern of the MFG depicted a progressive trend toward the long-chain glycerides (CAN 48 to 54), with a maximum at CAN 52, whereas the MFGM showed a bimodal trend with medium- and long-chain glycerides
(CAN 36 to 40), including 2 maxima at CAN 38 and 52. Significant differences were observed for each pair of CAN percentage values of the MFG and MFGM, except for CAN 42, 44, and 46, which reached the same values in both fractions.

Regarding the unsaturation degree of the fatty acids (FA; Figure 2A), the data showed that MUFA and PUFA had the same profiles in both the MFG and the MFGM fractions. Indeed, the long-chain FA occupied almost $90 \%$ of the FA profile in both fractions, although no significant differences were observed for either the unsaturation degree of the FA or their chain length, except for the very long-chain FA category, which was preferentially accumulated in MFGM. Published data regarding the saturated and unsaturated FA in human milk point to a different profile than the one found in this study. Jensen (1999) reviewed the FA composition of milk from women consuming Western and non-Western diets and found the fraction of saturated FA higher than that of the mono- and polyunsaturated FA in both diets, but here we observed the opposite result. Thus, 

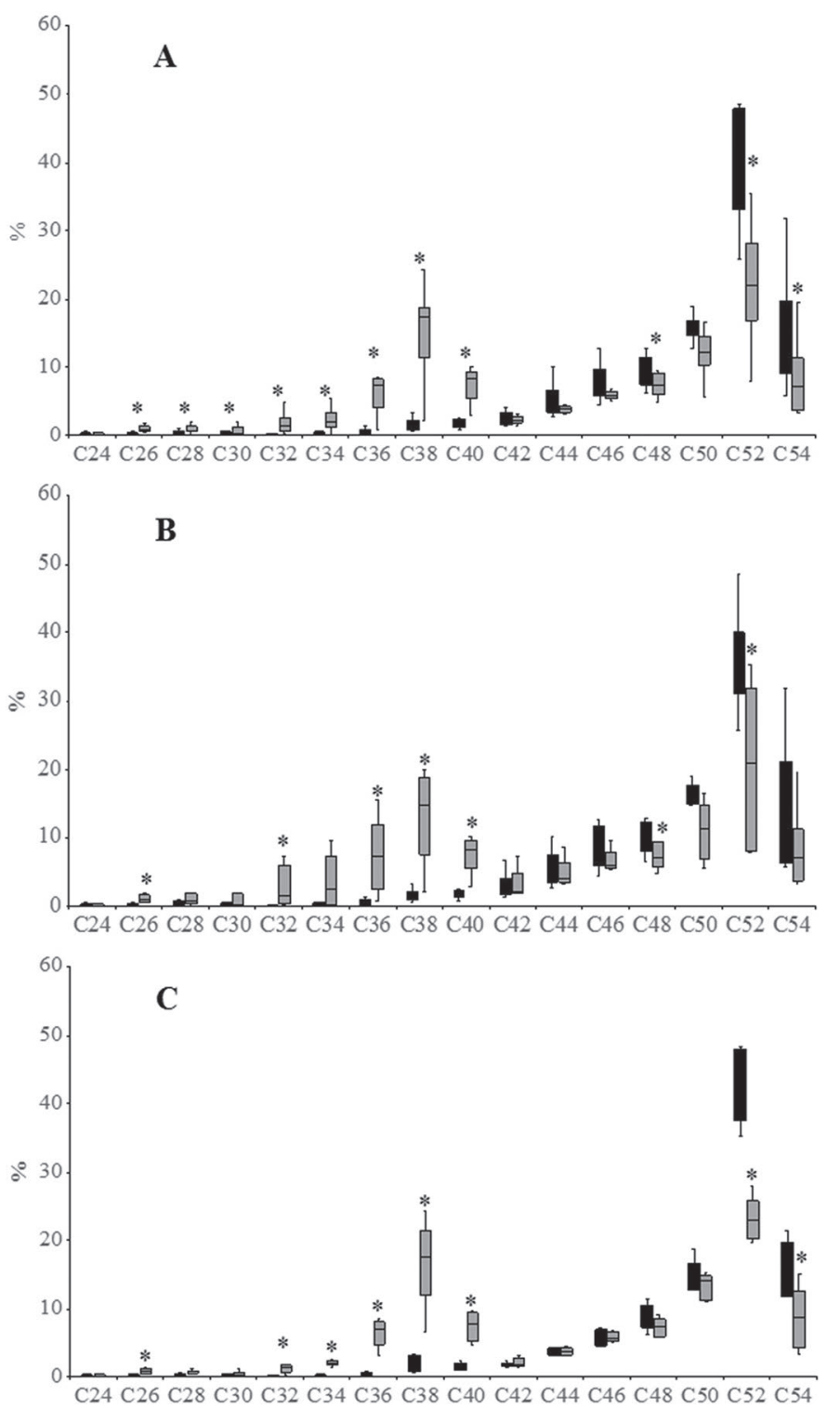

Figure 1. Chain length distribution (\%) of the triacylglycerides (TAG) in human colostrum samples according to their carbon atom numbers (CAN). Determinations were performed on the total milk fat fraction (black bars) and on the milk fat globule membrane fraction (gray bars). Significant differences between both determinations at each TAG CAN are denoted with an asterisk $(P<0.05)$. Data are presented for samples independent of gestational age (A), preterm human colostrum samples (B), or full-term human colostrum samples (C). Components in the box plots are as follows: midlines, median values; boxes, 75 th and 25 th percentile; bars, maximum and minimum values.

in that study, the ratio of saturated to unsaturated FA was 0.9 and 0.8 for human milk from Western and non-Western diets, respectively. Indeed, Yuhas et al. (2006) reported a similar value (0.8) for the FA profile of human milk from 8 countries. The value observed in our study with colostrum samples was 0.5. Thus, the milk from the mothers who participated in our study presented a higher proportion of PUFA (24.5\% median value, independent of gestational age) than the observed values in previous publications. The geographic location where our study was performed could facilitate a high consumption of fish and seafood products (100 $\mathrm{g}$ /person daily), which are significant dietary contributors to the PUFA content in milk (Varela-Moreiras et al., 2010). Another noteworthy result is that the evaluation of individual FA was independent of the location where they were determined (MFG or MFGM) except for the caprylic (C8:0), capric (C10:0), arachidonic (AA, C20:4), and docosahexaenoic (DHA, C22:6) FA. Hence, the contribution of these FA to the profile of the MFGM was significantly higher than in the MFG. Phospholipids and sphingolipids in the MFGM are highly unsaturated and saturated, respectively (Lopez et al., 2008; Abrahamse et al., 2012), and this combination of PL with soft versus tight-packed structure and low or high melting temperature is appropriate to maintain membrane structure and compartmentalization of membrane proteins (Evers et al., 2008; Gallier et al., 2010; Lopez et al., 2010; Lopez and Ménard, 2011; Zou et al., 2015; Nguyen et al., 2016).

The ratio of cholesterol to total amount of fat, expressed as percentage ratio (\% Chol/Fat, Figure 3A), was another quantity differently distributed in both MFG and MFGM scenarios, with a significantly higher proportion found in the MFGM. Cholesterol is a key component of cellular membranes, especially in the mammary cells (Keenan et al., 1970; Kanno et al., 1987), so the preferential accumulation of Chol in the MFGM was expected. Regarding the PL categories from both locations, they are minor constituents, although the contribution of the PL fraction in the MFGM to its total fat amount was almost 4-fold that of the MFG. The categories of PL of the MFG and the MFGM were qualitatively identical, but the quantitative distribution was different in each profile (Figure $4 \mathrm{~A})$. Thus, the profile of the MFG was dominated by SM $(36.2 \%)$, followed by similar proportions of PC and $\mathrm{PE}(29.1 \%$ and $27.8 \%$, respectively), and finally PS and PI with lower contributions. However, the PL in the MFGM were led by PC (37.6\%) and SM (27.7\%), then PE $(22.5 \%)$, and finally PS and PI. Comparison of the content (\%) of each category of PL in both MFG and MFGM locations yielded significant differences, except for PC. The chemical heterogenicity of PL in the membrane has been reported in milk from humans and other species of mammals (Keenan and Patton, 1995; Evers et al., 2008; Michalski, 2009; Claumarchirant et al., 2016). Our results are in the same trend as the data already published regarding the profile of PL in MFGM isolated from human milk; that is, PI and PS were the 

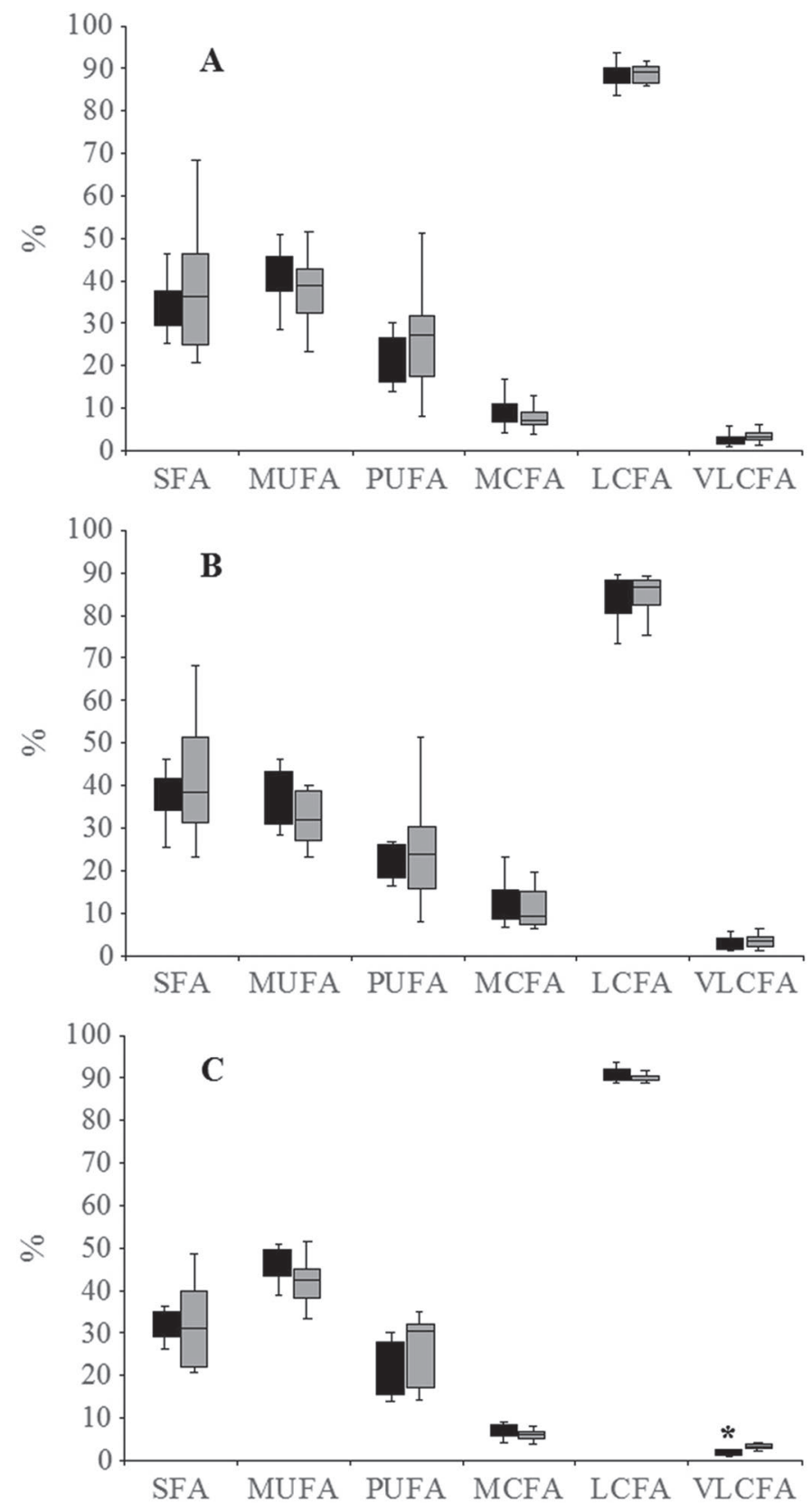

Figure 2. Features of fatty acids regarding their saturated or unsaturated profile and length of chain in all the human colostrum samples used in the study (A), preterm samples (B), or full-term samples (C). Determinations were performed on the milk fat globule (black bars) and on the milk fat globule membrane fraction (gray bars). MCFA = medium-chain fatty acids; LCFA $=$ long-chain fatty acids; VLCFA $=$ very long-chain fatty acids. Significant differences are noted with an asterisk $(P<0.05)$. Components in the box plots are as follows: midlines, median values; boxes, 75 th and 25 th percentile; bars, maximum and minimum values.

minor contributors, whereas PC, SM, and PE led the profile. However, the rank observed in our study for the 3 major contributors to the PL profile differed from the ranks reported in other studies (Lopez and Ménard,
2011; Claumarchirant et al., 2016). Changes in the rank may result from several factors influencing the pattern of human milk lipids (Harzer et al., 1983), including the collection of human milk samples at different lactation stages. Indeed, it is important to note that our study distinguished the PL in the MFGM from those measured in the MFG, so that differences may arise from additional stores of PL in the lipid core of the MFG.

\section{Changes of Lipid Classes in MFG and MFGM from Human Colostrum, According to Gestational Age}

Segregation of lipid families in the MFG is an outcome of the requirements for appropriate organizational and functional abilities of the MFGM, which is the original cellular plasma membrane of the mammary secretory epithelium. Therefore, most of the segregation of lipid categories observed here should arise from structural constraints operating at the mammary tissue, independent of its developing stage - basal, pregnancy, lactation, or involution (Inman et al., 2015). However, other lipid classes may differentiate between MFG and MFGM, resulting from the accretion of lipids to function as nutrients during lactation instead of any structural role at the mammary tissue. Hence, the analysis of lipid categories and the potential differences that could be observed between MFG and MFGM, considering the gestational age factor, was aimed to ascertain whether both kinds of lipid segregation (structural or nutritional) exist.

In the case of TAG, the trends of CAN distribution observed for MFG (monomodal) and MFGM (bimodal) were reproduced in both kinds of samples (Figures 1B and $1 \mathrm{C}$ ). Significant differences were conserved, independent of the delivery condition, except for $\mathrm{C} 48$ in the full-term samples and C54 in the preterm ones, which were equally distributed in MFG and MFGM fractions; however, the opposite result was observed for $\mathrm{C} 48$ and C54 in the full-term and preterm samples, respectively. Figures $3 \mathrm{~B}$ and $3 \mathrm{C}$ represent the $\% \mathrm{Chol} /$ Fat ratio values for MFG and MFGM in the preterm and fullterm colostrum samples. The segregation of cholesterol between both locations was independent of delivery age (Figure 3A), with significant differences observed in both types of samples. However, if we analyze the increment of the \% Chol/Fat values in MFGM to those in MFG, full-term samples showed significantly higher increases compared with those observed in preterm samples. According to these results, the CAN distribution of TAG and the gradient of cholesterol from the core to the envelope of the MFG should be considered structural segregations, although some dynamics still occurred for Chol and specific long-chain TAG during 

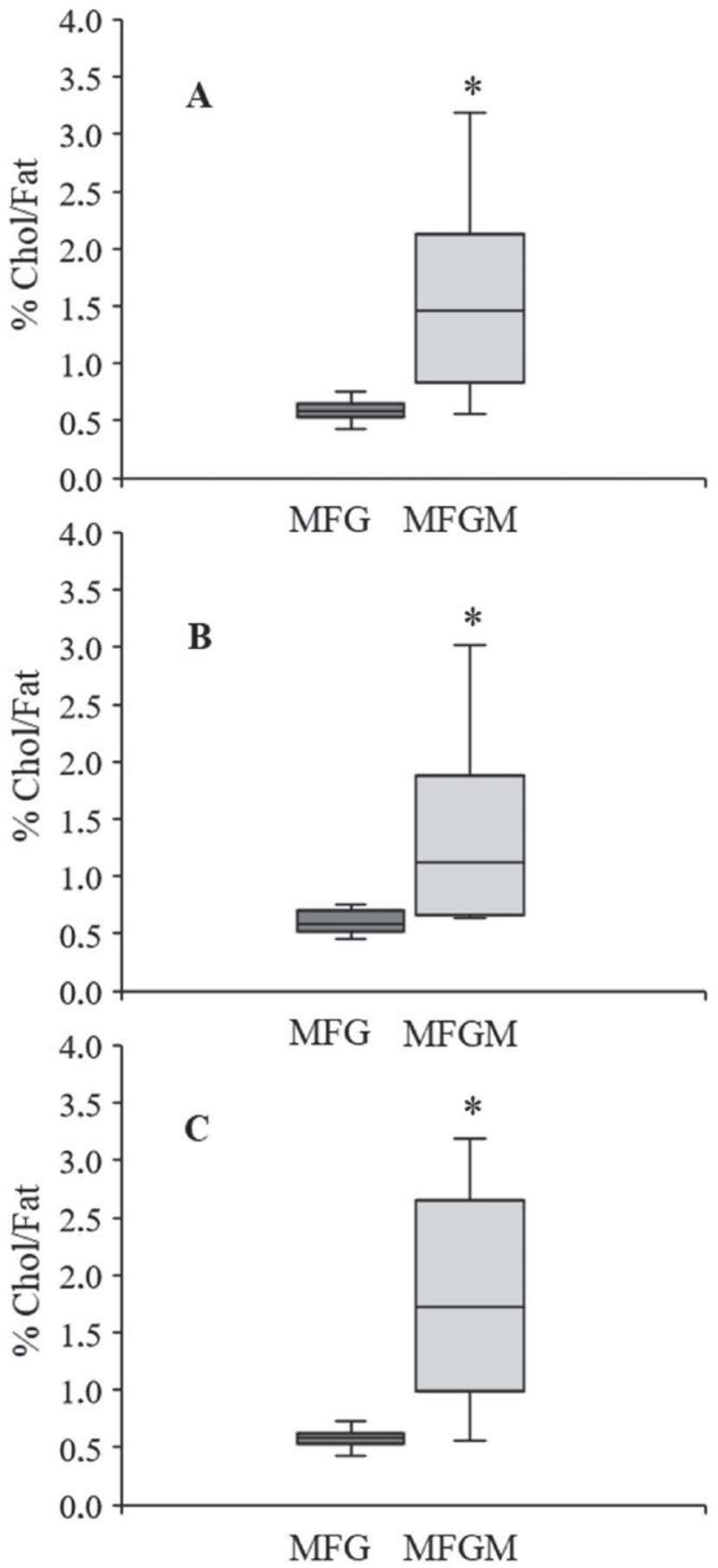

Figure 3. Ratio of cholesterol to total fat (\% Chol/Fat) in human colostrum samples. Determinations were performed on the total milk fat fraction (MFG, dark gray bars), and in the milk fat globule membrane fraction (MFGM, light gray bars). Significant differences between the determinations at each location are denoted with an asterisk $(P<0.05)$. Data are presented for samples independent of gestational age (A), preterm human colostrum samples (B), and full-term human colostrum samples (C). No significant differences were observed when the $\% \mathrm{Chol} /$ Fat ratio values were compared for the same fraction (MFG or MFGM), but differences were detected at different gestational ages (preterm or full term). Components in the box plots are as follows: midlines, median values; boxes, 75 th and 25 th percentile; bars, maximum and minimum values. the last weeks of pregnancy. Regarding the unsaturation degree of the FA, preterm versus full-term delivery was not a significant factor, and the data were distributed in the same fashion as in Figure 2A; that is, MUFA and PUFA led the profile in both the MFG and MFGM fractions (Figures 2B and 2C). Indeed, the long-chain FA represented almost $90 \%$ of the FA profile in both fractions, whereas no significant differences were observed for either the unsaturation degrees of FA or their chain length, except for the very long-chain FA category, which was preferentially accumulated in the MFGM. Additionally, individual FA distribution was also independent of gestational age at delivery. Therefore, the conclusions previously discussed also applied if data were evaluated considering gestational age.

The distribution of PL categories in MFG and MFGM colostrum was also dependent on gestational age, as can be observed in Figures $4 \mathrm{~B}$ and $4 \mathrm{C}$. Hence, although the rankings for PL families in the MFG and MFGM fractions were independent of gestational age $(\mathrm{SM}>\mathrm{PC}>\mathrm{PE}>\mathrm{PS}>\mathrm{PI}$ in $\mathrm{MFG}$, and $\mathrm{PC}>\mathrm{SM}$ $>\mathrm{PE}>\mathrm{PS}>\mathrm{PI}$ in MFGM), when occurrence of the PL categories in both fractions was compared individually, diverse trends were observed, regarding the significant differences among them. Phosphatidylcholine (PC) was consistently distributed in both the MFG and the MFGM; that is, no segregation of this lipid class occurred, and independent of gestational age, no significant differences were observed between fractions. Indeed, PS and PI showed significantly greater contributions to the profile of PL of the MFGM, independent of gestational age. However, when presence of $\mathrm{PE}$ was compared, significant differences were observed only in preterm samples, whereas occurrence of SM was significantly different between both fractions only in full-term samples.

This comparative analysis led to noteworthy outcomes about the changing role of the PL, considering gestational age, and whether they were segregated in the MFG. Holmes-McNary et al. (1996) show that concentration of choline-containing lipids is not associated with gestational age. In this study we showed that the lack of correlation of PC with delivery term was also independent of the location of the MFG where it was measured. Therefore, PC could be denoted as a structural category, in correspondence with its critical role in the biophysics of cellular membranes. The same conclusion could be inferred for PS and PI. Their higher contribution to the PL profile when they were determined in the MFGM fraction, and that this segregation was independent of the gestational age condition, denoted both PL as structural categories in the MFGM. However, SM and PE deserve attention, as their contribution to the PL profile tended to decrease 


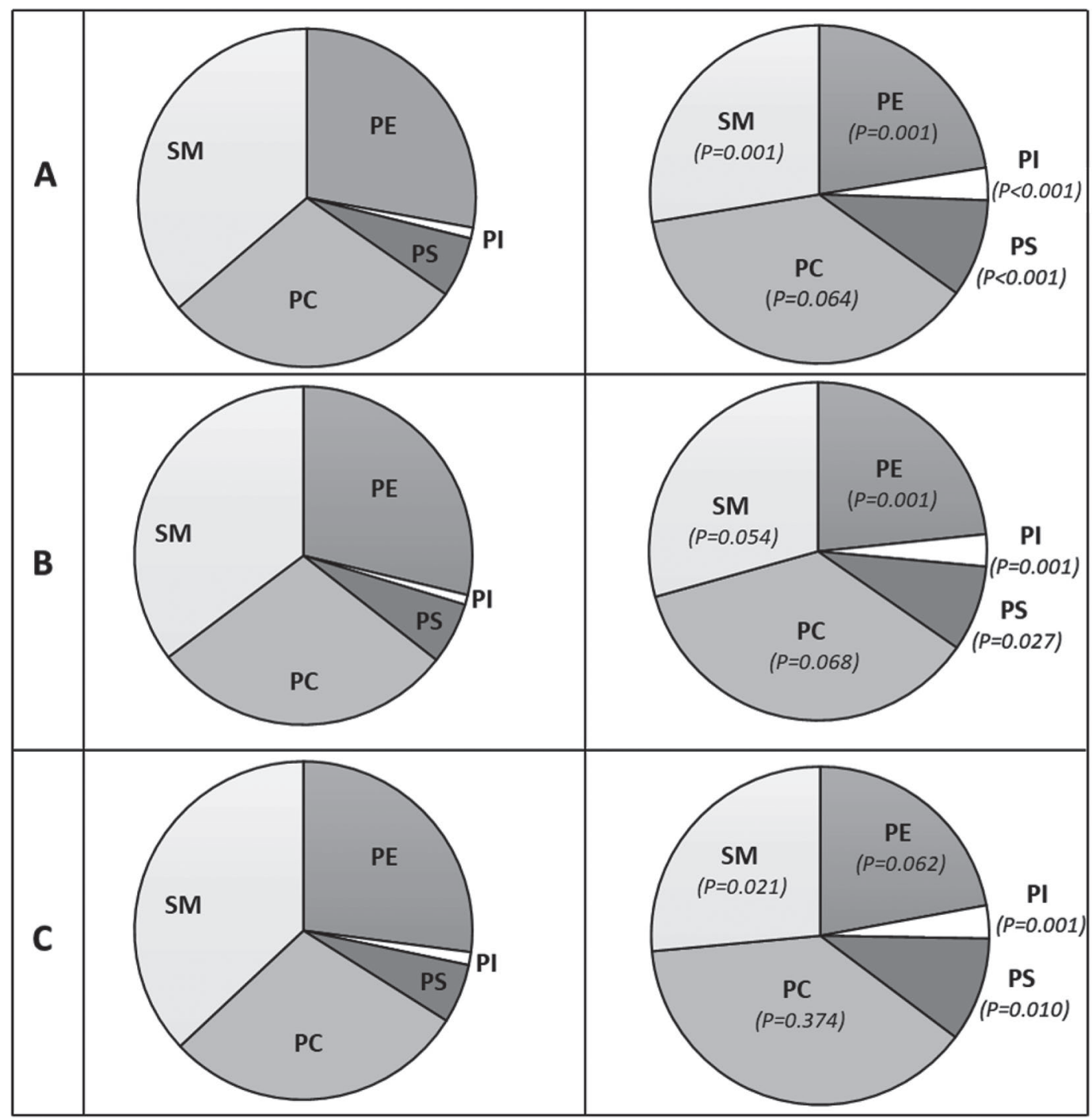

Figure 4. Distribution of polar lipid (PL) categories (\%) in human colostrum samples. Determinations were performed on the total milk fat fraction (MFG, left-hand column) and on the milk fat globule membrane fraction (MFGM, right-hand column). Paired comparisons of the individual PL categories between both fractions (MFG and MFGM) were made. $P<0.05$ was declared statistically significant. Data are presented for samples independent of gestational age (A), only preterm human colostrum samples (B), or only full-term human colostrum samples (C). SM = sphingomyelin; $\mathrm{PE}=$ phosphatidylethanolamine; $\mathrm{PI}=$ phosphatidylinositol; $\mathrm{PS}=$ phosphatidylserine; $\mathrm{PC}=$ phosphatidylcholine

when they were measured in the MFGM, although significant differences were established only in the fullterm samples for SM. This difference might indicate that, in the final stage of pregnancy, specific accretion of some PL occurred but in a different location than the MFGM. This hypothesis has been suggested by some authors who point to the existence of aggregates sourcing PL (Gallier et al., 2010; Blans et al., 2017), which are only separated in MFG and MFGM after the application of high-ultracentrifugation steps. Therefore, such differences correlated with the gestational age condition, it has been proved, should be denoted as nutritional redistribution but not associated with the functional and operational capabilities of the MFGM, for either preterm or full-term delivery.

\section{ACKNOWLEDGMENTS}

This study was supported in part by the Spanish Ministry of Science, Innovation and Universities (Project AGL2017-87884; MINECO/AEI/FEDER, UE; Madrid, Spain). The authors are grateful to the lactating mothers who donate milk at the Hospital Virgen del Rocío de Sevilla (Seville, Spain). Molly J. Davis (The Ohio State University, Columbus) edited and proofread the paper. The authors declare no conflict of interest. 


\section{REFERENCES}

Abrahamse, E., M. Minekus, G. A. van Aken, B. van de Heijning, J. Knol, N. Bartke, R. Oozeer, E. M. van der Beek, and T. Ludwig. 2012. Development of the digestive system-experimental challenges and approaches of infant lipid digestion. Food Dig. 3:63-77. https: //doi.org/10.1007/s13228-012-0025-x.

Bitman, J., D. L. Wood, M. Hamosh, P. Hamosh, and N. Mehta. 1983. Comparison of the lipid composition of breast milk from mothers of term and preterm infants. Am. J. Clin. Nutr. 38:300-312. https: //doi.org/10.1093/ajen/38.2.300.

Blans, K., M. S. Hansen, L. V. Sorensen, M. L. Hvam, K. A. Howard, A. Moller, L. Wiking, L. B. Larsen, and J. T. Rasmussen. 2017. Pellet-free isolation of human and bovine milk extracellular vesicles by size-exclusion chromatography. J. Extracell. Vesicles 6:1294340. https://doi.org/10.1080/20013078.2017.1294340.

Bokor, S., B. Koletzko, and T. Decsi. 2007. Systematic review of fatty acid composition of human milk from mothers of preterm compared to full-term infants. Ann. Nutr. Metab. 51:550-556. https:/ /doi.org/10.1159/000114209.

Calvo, M. V., M. C. Martín-Hernández, A. García-Serrano, M. P. Castro-Gómez, L. Alonso-Miravalles, R. García-Martín, J. MeginoTello, L. Alonso, and J. Fontecha. 2020. Comprehensive characterization of neutral and polar lipids of buttermilk from different sources and its milk fat globule membrane isolates. J. Food Compos. Anal. 86:103386. https://doi.org/10.1016/j.jfca.2019.103386.

Castro-Gómez, M. P., J. Fontecha, and L. M. Rodríguez-Alcalá. 2014b. A high-performance direct transmethylation method for total fatty acids assessment in biological and foodstuff samples. Talanta 128:518-523. https://doi.org/10.1016/j.talanta.2014.05.051.

Castro-Gómez, M. P., L. M. Rodriguez-Alcalá, M. V. Calvo, J. Romero, J. A. Mendiola, E. Ibañez, and J. Fontecha. 2014a. Total milk fat extraction and quantification of polar and neutral lipids of cow, goat, and ewe milk by using a pressurized liquid system and chromatographic techniques. J. Dairy Sci. 97:6719-6728. https:// doi.org/10.3168/jds.2014-8128.

Cilla, A., K. D. Quintaes, R. Barberá, and A. Alegría. 2016. Phospholipids in human milk and infant formulas: Benefits and needs for correct infant nutrition. Crit. Rev. Food Sci. Nutr. 56:1880-1892. https://doi.org/10.1080/10408398.2013.803951.

Claumarchirant, L., A. Cilla, E. Matencio, L. M. Sanchez-Siles, P. Castro-Gomez, J. Fontecha, A. Alegría, and M. J. Lagarda. 2016. Addition of milk fat globule membrane as an ingredient of infant formulas for resembling the polar lipids of human milk. Int. Dairy J. 61:228-238. https://doi.org/10.1016/j.idairyj.2016.06.005.

Deeth, H. C. 1997. The role of phospholipids in the stability of milk fat globules. Aust. J. Dairy Technol. 52:44-46.

Evers, J. M., R. G. Haverkamp, S. E. Holroyd, G. B. Jameson, D. D. S. Mackenzie, and O. J. McCarthy. 2008. Heterogeneity of milk fat globule membrane structure and composition as observed using fluorescence microscopy techniques. Int. Dairy J. 18:1081-1089. https://doi.org/10.1016/j.idairyj.2008.06.001.

Gallier, S., D. Gragson, C. Cabral, R. Jiménez-Flores, and D. W. Everett. 2010. Composition and fatty acid distribution of bovine milk phospholipids from processed milk products. J. Agric. Food Chem. 58:10503-10511. https://doi.org/10.1021/jf101878d.

German, J. B., and C. J. Dillard. 2006. Composition, structure and absorption of milk lipids: A source of energy, fat-soluble nutrients and bioactive molecules. Crit. Rev. Food Sci. Nutr. 46:57-92. https://doi.org/10.1080/10408690590957098.

Harzer, G., M. Haug, I. Dieterich, and P. Gentner. 1983. Changing patterns of human milk lipids in the course of lactation and during the day. Am. J. Clin. Nutr. 37:612-621. https://doi.org/10.1093/ ajcn/37.4.612.

Holmes-McNary, M. Q., W.-L. Cheng, M.-H. Mar, S. Fussell, and S. H. Zeisel. 1996. Choline and choline esters in human and rat milk and in infant formulas. Am. J. Clin. Nutr. 64:572-576. https://doi .org/10.1093/ajcn/64.4.572.

Ingvordsen Lindahl, I. E., V. M. Artegoitia, E. Downey, J. A. O'Mahony, C.-A. O'Shea, C. A. Ryan, A. L. Kelly, H. C. Bertram, and U. K. Sundekilde. 2019. Quantification of human milk phos- pholipids: The effect of gestational and lactational age on phospholipid composition. Nutrients 11:222. https://doi.org/10.3390/ nu11020222.

Inman, J. L., C. Robertson, J. D. Mott, and M. J. Bissell. 2015. Mammary gland development: Cell fate specification, stem cells and the microenvironment. Development 142:1028-1042. https://doi.org/ 10.1242/dev.087643.

Jensen, R. G. 1989. The Lipids of Human Milk. CRC Press, Boca Raton, FL.

Jensen, R. G. 1999. Lipids in human milk. Lipids 34:1243-1271. https: //doi.org/10.1007/s11745-999-0477-2.

Julious, S. A., and R. J. Owen. 2006. Sample size calculations for clinical studies allowing for uncertainty about the variance. Pharm. Stat. 5:29-37. https://doi.org/10.1002/pst.197.

Kanno, C., H. Hattori, and K. Yamauchi. 1987. Lipid composition of plasma membranes isolated from lactating bovine mammary gland. Agric. Biol. Chem. (Japan) 51:2995-3001.

Keenan, T. W., and I. H. Mather. 2006. Intracellular origin of milk fat globules and the nature of the milk fat globule membrane. Pages 137-171 in Advanced Dairy Chemistry, Vol. 2: Lipids. 3rd ed. P. F. Fox and P. L. H. McSweeney, ed. Springer, New York, NY.

Keenan, T. W., D. J. Morre', D. E. Olson, W. N. Yunghans, and S. Patton. 1970. Biochemical and morphological comparison of plasma membrane and milk fat globule membrane from bovine mammary gland. J. Cell Biol. 44:80-93. https://doi.org/10.1083/ jcb.44.1.80.

Keenan, T. W., and S. Patton. 1995. The structure of milk: Implications for sampling and storage. Pages 5-50 in Handbook of Milk Composition. R. G. Jensen, ed. Academic Press, Cambridge, MA.

Kramer, M. S., and R. Kakuma. 2012. Optimal duration of exclusive breastfeeding. Cochrane Database Syst. Rev. CD003517. https:// doi.org/10.1002/14651858.CD003517.

Lee, H., E. Padhi, Y. Hasegawa, J. Larke, M. Parenti, A. Wang, O. Hernell, B. Lönnerdal, and C. Slupsky. 2018. Compositional dynamics of the milk fat globule and its role in infant development. Front Pediatr. 6:313. https://doi.org/10.3389/fped.2018.00313.

Löfgren, L., M. Ståhlman, G. Forsberg, S. Saarinen, R. Nilsson, and G. I. Hansson. 2012. The BUME method: A novel automated chloroform-free 96-well total lipid extraction method for blood plasma. J. Lipid Res. 53:1690-1700. https://doi.org/10.1194/jlr.D023036.

Lopez, C. 2011. Milk fat globules enveloped by their biological membrane: Unique colloidal assemblies with a specific composition and structure. Curr. Opin. Colloid Interface Sci. 16:391-404. https:// doi.org/10.1016/j.cocis.2011.05.007.

Lopez, C., V. Briard-Bion, O. Menard, F. Rousseau, P. Pradel, and J.-M. Besle. 2008. Phospholipid, sphingolipid, and fatty acid compositions of the milk fat globule membrane are modified by diet. J. Agric. Food Chem. 56:5226-5236. https://doi.org/10.1021/ jf7036104.

Lopez, C., C. Cauty, and F. Guyomarc'h. 2019. Unraveling the complexity of milk fat globules to tailor bioinspired emulsions providing health benefits: The key role played by the biological membrane. Eur. J. Lipid Sci. Technol. 121:1800201.

Lopez, C., M. N. Madec, and R. Jimenez-Flores. 2010. Lipid rafts in the bovine milk fat globule membrane revealed by the lateral segregation of phospholipids and heterogeneous distribution of glycoproteins. Food Chem. 120:22-33. https://doi.org/10.1016/j .foodchem.2009.09.065

Lopez, C., and O. Ménard. 2011. Human milk fat globules: Polar lipid composition and in situ structural investigations revealing the heterogeneous distribution of proteins and the lateral segregation of sphingomyelin in the biological membrane. Colloids Surf. B Biointerfaces 83:29-41. https://doi.org/10.1016/j.colsurfb.2010.10.039.

Mather, I. H. 2000. A review and proposed nomenclature for major proteins of the milk-fat globule membrane. J. Dairy Sci. 83:203247. https://doi.org/10.3168/jds.S0022-0302(00)74870-3.

McManaman, J. L., and M. C. Neville. 2003. Mammary physiology and milk secretion. Adv. Drug Deliv. Rev. 55:629-641. https://doi .org/10.1016/S0169-409X(03)00033-4.

McManaman, J. L., M. E. Reyland, and E. C. Thrower. 2006. Secretion and fluid transport mechanisms in the mammary gland: 
Comparisons with the exocrine pancreas and the salivary gland. J. Mammary Gland Biol. Neoplasia 11:249-268. https://doi.org/10 $.1007 / \mathrm{s} 10911-006-9031-3$.

Michalski, M.-C. 2009. Specific molecular and colloidal structures of milk fat affecting lipolysis, absorption and postprandial lipemia. Eur. J. Lipid Sci. Technol. 111:413-431. https://doi.org/10.1002/ ejlt.200800254.

Nguyen, H. T. H., M.-N. Madec, L. Ong, S. E. Kentish, S. L. Gras, and C. Lopez. 2016. The dynamics of the biological membrane surrounding the buffalo milk fat globule investigated as a function of temperature. Food Chem. 204:343-351. https://doi.org/10.1016/j foodchem.2016.02.141.

Ortega-Anaya, J., and R. Jimenez-Flores. 2019. Symposium review: The relevance of bovine milk phospholipids in human nutritionEvidence of the effect on infant gut and brain development. J. Dairy Sci. 102:2738-2748. https://doi.org/10.3168/jds.2018-15342.

Patton, S., and G. E. Huston. 1986. A method for isolation of milk fat globules. Lipids 21:170-174. https://doi.org/10.1007/BF02534441.

Shoji, H., T. Shimizu, N. Kaneko, K. Shinohara, S. Shiga, M. Saito, K. Oshida, T. Shimizu, M. Takase, and Y. Yamashiro. 2006. Com- parison of the phospholipid classes in human milk in Japanese mothers of term and preterm infants. Acta Paediatr. 95:996-1000. https://doi.org/10.1080/08035250600660933.

Varela-Moreiras, G., J. M. Ávila, C. Cuadrado, S. del Pozo, E. Ruiz, and O. Moreiras. 2010. Evaluation of food consumption and dietary patterns in Spain by the Food Consumption Survey: Updated information. Eur. J. Clin. Nutr. 64(Suppl. 3):S37-S43. https: //doi.org/10.1038/ejcn.2010.208.

Xavier, A. A. O., E. Díaz-Salido, I. Arenilla-Vélez, J. Aguayo-Maldonado, J. Garrido-Fernández, J. Fontecha, A. Sánchez-García, and A. Pérez-Gálvez. 2018. Carotenoid content in human colostrum is associated to preterm/full-term birth condition. Nutrients 10:1654. https://doi.org/10.3390/nu10111654.

Yuhas, R., K. Pramuk, and E. L. Lien. 2006. Human milk fatty acid composition from nine countries varies most in DHA. Lipids 41:851-858. https://doi.org/10.1007/s11745-006-5040-7.

Zou, X., Z. Guo, Q. Jin, J. Huang, L. Cheong, X. Xu, and X. Wang. 2015. Composition and microstructure of colostrum and mature bovine milk fat globule membrane. Food Chem. 185:362-370. https://doi.org/10.1016/j.foodchem.2015.03.145. 